\title{
Role of Subsurface Oxygen in Oxide Formation at Transition Metal Surfaces
}

\author{
M. Todorova, ${ }^{1}$ W. X. Li, ${ }^{1}$ M. V. Ganduglia-Pirovano, ${ }^{1, *}$ C. Stampfl, ${ }^{1,2}$ K. Reuter,${ }^{1}$ and M. Scheffler ${ }^{1}$ \\ ${ }^{1}$ Fritz-Haber-Institut der Max-Planck-Gesellschaft, Faradayweg 4-6, D-14195 Berlin, Germany \\ ${ }^{2}$ Northwestern University, 2145 Sheridan Road, Evanston Illinois60208
}

(Received 3 December 2001; published 12 August 2002)

\begin{abstract}
We present a density-functional theory trend study addressing the incorporation of oxygen into the basal plane of the late $4 d$ transition metals (TMs) from Ru to Ag. Occupation of subsurface sites is always connected with a significant distortion of the host lattice, rendering it initially less favorable than onsurface chemisorption. Penetration starts only after a critical coverage $\theta_{c}$, which is lower for the softer metals towards the right of the TM series. The computed $\theta_{c}$ are found to be very similar to those above which the bulk oxide phase becomes thermodynamically more stable, thus suggesting that the initial incorporation of $\mathrm{O}$ actuates the formation of a surface oxide on TM surfaces.
\end{abstract}

PACS numbers: 81.65.Mq, 68.43.Bc, 68.43.-h

The use of transition metals (TMs) in oxidation catalysts has motivated a large number of studies on oxygen-metal interactions at the low index surfaces of materials such as $\mathrm{Rh}, \mathrm{Pd}$, and Pt. Although the technical reactions proceed at rather oxidizing conditions with atmospheric pressures and elevated temperatures, microscopic modeling typically concentrated on the adsorption and oxidation of reactants at the surface of more or less ideal metal substrates [1]. A possible oxidation of the surface in a reactive environment is sometimes perceived as leading to an inactive surface oxide layer poisoning the catalytic reaction. That oxide patches may instead also be the active centers on the surface has only recently been indicated in experiments addressing the $\mathrm{CO}$ oxidation over $\mathrm{Ru}(0001)$ and $\mathrm{Pt}(110)$ surfaces [2,3].

These findings call for a deeper, atomistic understanding of the oxidation of TM surfaces, in particular, to investigate the ease of (or resistance to) oxide formation that could then become relevant in catalytic applications. Unfortunately, this understanding is still very shallow for everything that goes beyond on-surface chemisorption of $\mathrm{O}$ at low coverages. King and co-workers [4] proposed that the critical coverage, $\theta_{c}^{\text {thd }}$, at which the transition between a chemisorbed phase and the appearance of an oxidic film occurs is thermodynamically, not kinetically, determined. Below $\theta_{c}^{\text {thd }}$, the heat of formation of the chemisorbed phase is higher than the heat of formation of the oxide. Repulsive interactions between the $\mathrm{O}$ adatoms drive the differential heat of adsorption down sharply with increasing coverage, until at $\theta_{c}^{\text {thd }}$ it is equal to that of the formation of an oxide film.

While this model has already been successfully applied to explain experimental data on the critical coverage for oxide formation on $\mathrm{Ni}(100)$ and $\operatorname{Ag}(111)$ [4], it does not account for the microscopic processes underlying the phase transition from the two-dimensional adsorbate layer to the three-dimensional oxide. To this end, we have performed density-functional theory (DFT) calculations addressing the initial incorporation of $\mathrm{O}$ into the basal surface of the late $4 d \mathrm{TMs}$ from $\mathrm{Ru}$ to $\mathrm{Ag}$. We find that incorporation of $\mathrm{O}$ into the subsurface region is initially always less stable than on-surface chemisorption, largely due to the additional cost of distorting the substrate lattice and breaking metal bonds. With increasing on-surface coverage, this preference decreases due to repulsive interactions within the more densely packed electronegative $\mathrm{O}$ adlayer. Above a certain critical $O$ coverage, $\theta_{c}$, occupation of subsurface sites then becomes more favorable compared to a continued filling of the on-surface sites. The softer the material, the lower the $\theta_{c}$ : Incorporation commences only after completion of a full monolayer (ML) on the surface for the case of $\mathrm{Ru}$, but already at low on-surface coverages for the case of Ag. The obtained $\theta_{c}$ are very similar to the critical coverages for oxide formation, $\theta_{c}^{\text {thd }}$, obtained with King's thermodynamic model. This leads us to suggest that the initial incorporation of subsurface $\mathrm{O}$ is the "bottleneck" in the oxidation sequence of these metal surfaces. As we show this incorporation to be strongly dependent on lattice deformation properties, the ease of oxide formation will then follow similar trends as the bulk cohesive energy or the bulk modulus.

In our DFT calculations, we employ the generalized gradient approximation for the exchange-correlation functional [5]. The Kohn-Sham equations are solved using the full-potential linearized augmented plane wave (FPLAPW) method for the elements $\mathrm{Ru}, \mathrm{Rh}$, and Pd [6], and the pseudopotential approach for $\mathrm{Ru}$ and $\mathrm{Ag}$ [7]. The highly converged basis sets of our supercell calculations [8-12] ensure the determination of relative binding energy differences, which are the central quantities obtained from the computations, within a numerical uncertainty of $\pm 50 \mathrm{meV}$.

The oxidation of TMs commences with the dissociative chemisorption of $\mathrm{O}_{2}$ at highly coordinated sites on the surface, possibly preceded by molecular physisorption at low temperatures. To address the on-surface $\mathrm{O}$ adsorption at sub-ML coverages, $0<\theta \leq 1 \mathrm{ML}$, for the late $4 d \mathrm{TM}$ sequence from $\mathrm{Ru}$ to $\mathrm{Ag}$, we employ $(2 \times 2)$ unit cells and 
calculate structures containing from one $(\theta=0.25 \mathrm{ML})$ up to four $(\theta=1 \mathrm{ML})$ on-surface $\mathrm{O}$ atoms. On the considered basal surface, we find that $\mathrm{O}$ always favors adsorption in the hollow sites, which represent a continuation of the bulk stacking sequence, i.e., hcp sites on $\mathrm{Ru}(0001)$ and fcc sites on $\operatorname{Rh}(111), \operatorname{Pd}(111)$, and $\operatorname{Ag}(111)$ [9-12]. The absolute values of the corresponding on-surface average binding energies $E_{b}$, shown in Fig. 1, decrease progressively for the elements towards the right of the periodic table, which is due to the continued filling of the $d$ band in the late TMs, leading to an increased occupation of antibonding oxygenmetal states [13].

Additionally, we observe a marked decrease of $E_{b}$ with coverage, which reflects a repulsive interaction within the more and more densely packed electronegative overlayer. This decrease in $E_{b}$ translates into a steeply decreasing differential heat of adsorption, $\partial H_{\mathrm{ad}} / \partial \theta$, which we can compare with the experimental heat of formation of the most stable bulk oxide phase of the respective metal, $\Delta H_{f}$ [14]. Following King's thermodynamic argument [4], the critical on-surface coverage for the transition to the oxide phase, $\theta_{c}^{\text {thd }}$, is given once the differential heat of adsorption has fallen below the value of $\Delta H_{f}$. Using the computed $E_{b}$ and $\partial E_{b} / \partial \theta$ shown in Fig. 1, we evaluate $\Delta H_{f}=\left[\partial H_{\text {ad }} / \partial \theta\right]_{\theta_{c}^{\text {thd }}} \approx\left[E_{b}+\theta\left(\partial E_{b} / \partial \theta\right)\right]_{\theta_{c}^{\text {thd }}}$ to obtain $\theta_{c}^{\text {thd }}(\mathrm{Ru})=0.89 \mathrm{ML}, \quad \theta_{c}^{\text {thd }}(\mathrm{Rh})=0.59 \mathrm{ML}, \quad \theta_{c}^{\text {thd }}(\mathrm{Pd})=$ $0.50 \mathrm{ML}$, and $\theta_{c}^{\text {thd }}(\mathrm{Ag})=0.24 \mathrm{ML}$. Therefore, within this model, for Ru the bulk oxide phase is expected to become only more stable after almost a full ML of O on the surface, while for Rh and Pd this value sequentially decreases, until it is less than a quarter of a ML for Ag. We note in passing that, already at this stage, the considered TM surfaces exhibit a fundamentally different oxidation behavior compared to a simple $s p$ metal such as Al. For the latter, attractive interactions among the on-surface $\mathrm{O}$ adsorbates [15] lead to the formation of close-packed islands already at low coverages [16]. While the same thermodynamic

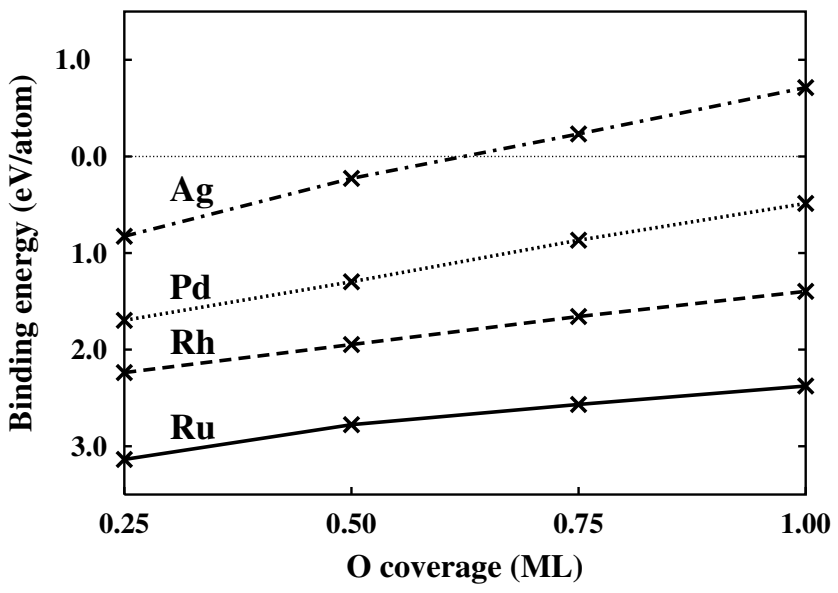

FIG. 1. Calculated average binding energies, $E_{b}$, for on-surface $\mathrm{O}$ chemisorption at the basal surface of the late $4 d$ TMs. The energies are given with respect to $\frac{1}{2} \mathrm{O}_{2}$. reasoning is, of course, applicable also, the chemical potential is in the latter case no longer determined by the differential heat of adsorption, but by the $E_{b}$ of $\mathrm{O}$ in the $(1 \times 1)$ phase, corresponding to $O$ removal from kink sites at the perimeter of sufficiently large adsorbate islands. Employing the numbers reported, e.g., in Ref. [15], we thus see that oxide formation on $\mathrm{Al}(111)$ may thermodynamically set in already at the lowest coverages, in agreement with experimental findings [16].

Trying to supplement the thermodynamic trend with microscopic information, we next address the incorporation of the first $\mathrm{O}$ atom into the subsurface region of the TM surface, which initiates the transition from the two-dimensional overlayer to a three-dimensional oxide nucleus. In order to assess at what critical coverage, $\theta_{c}$, $\mathrm{O}$ incorporation into the metal sets in, we again employ $(2 \times 2)$ unit cells and calculate surface structures containing from one $(\theta=0.25 \mathrm{ML})$ up to four $(\theta=1 \mathrm{ML}) \mathrm{O}$ atoms. Of these $N_{\text {tot }} \mathrm{O}$ atoms, $0 \leq N_{\text {sub }} \leq N_{\text {tot }}$ can be located below the surface, which defines the subsurface fraction $N_{\text {sub }} / N_{\text {tot }}$. The occupation of subsurface sites will in this simple model commence, when at the same total coverage, $\theta$, a structure with $N_{\text {sub }} / N_{\text {tot }}>0 \%$ is more stable than the hitherto discussed pure on-surface adsorption corresponding to $N_{\text {sub }} / N_{\text {tot }}=0 \%$.

We consider incorporation into all three high-symmetry interstitial sites between the first and second metal layer, namely, the octahedral (octa) and the tetrahedral (tetra-I) site directly below the on-surface fcc and hcp site, respectively, as well as a second tetrahedral (tetra-II) site directly below a first layer metal atom. For every given $\left(\theta, N_{\text {sub }} / N_{\text {tot }}\right)$, this implies that we have to test structures with all possible site combinations of the $\left(N_{\text {tot }}-N_{\text {sub }}\right)$ onsurface $\mathrm{O}$ in either hcp or fcc sites, and the $N_{\text {sub }}$ subsurface $\mathrm{O}$ in either of the three interstitial sites. We note that for some coverages this means that as many as 12 trial structures may result for just one $\left(\theta, N_{\text {sub }} / N_{\text {tot }}\right)$ point. We always find the combination on-surface $\mathrm{O}$ in fcc sites and subsurface $\mathrm{O}$ in tetra-I sites to be either most stable or energetically very close to the most stable geometry, which is why we will concentrate on this structural combination in the following.

For $\mathrm{O}$ in fcc sites on the surface and in tetra-I sites below, Fig. 2 shows the computed $E_{b}$ contours for each of the four elements. We see that, for $\mathrm{Ru}$ and $\mathrm{Rh}$, the pure on-surface chemisorption $\left(N_{\text {sub }} / N_{\text {tot }}=0 \%\right)$ is always the most favorable in the whole sub-ML coverage range considered in this work; i.e., O incorporation will start only after completion of a full $\mathrm{O}(1 \times 1)$ adlayer on the surface, $\theta_{c}>$ $1 \mathrm{ML}$, as shown in previous work addressing the coverage range $1 \mathrm{ML}<\theta \leq 3 \mathrm{ML}[10,11]$ and in agreement with experimental findings $[17,18]$. Still, we notice that the shallower valley in the Rh contour plot might account for a small, but finite concentration of subsurface $\mathrm{O}$ already at total coverages slightly below $1 \mathrm{ML}$ at elevated temperatures [19]. For Pd, $\theta_{c}$ has decreased well below $1 \mathrm{ML}$, and 


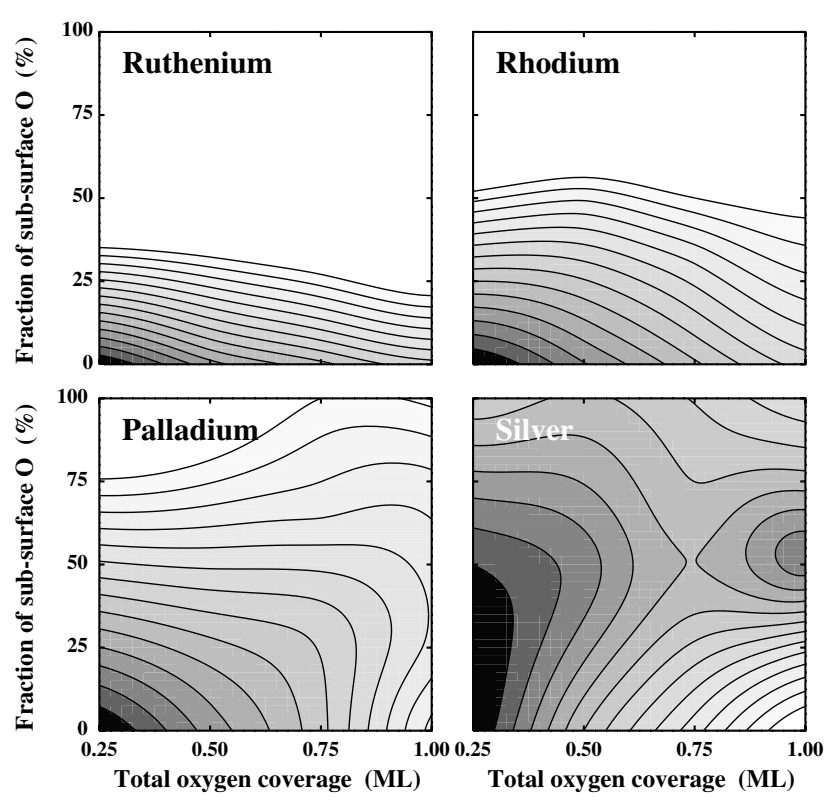

FIG. 2. Average binding energy $E_{b}$ as a function of the total $\mathrm{O}$ coverage of which a certain fraction $N_{\text {sub }} / N_{\text {tot }}$ is located below the surface (on-surface in fcc and subsurface in tetra-I sites). The highest $E_{b}$ are always found for the pure on-surface chemisorption phase at $\theta=0.25 \mathrm{ML}$ (chosen as zero reference, black area), with each contour line (lighter gray areas) at $0.1 \mathrm{eV}$ steps towards less stable $E_{b}$.

this lowering of $\theta_{c}$ continues for Ag for which only a small on-surface coverage may be stabilized before $\mathrm{O}$ goes subsurface. Again, both $\theta_{c}$ are in reasonable agreement with experiment, which finds initial surface oxides at $\operatorname{Pd}(111)$ and $\operatorname{Ag}(111)$ with local coverages of $\sim 0.6 \mathrm{ML}$ and $\sim 0.4 \mathrm{ML}$, respectively $[4,20]$. This gives us confidence that we capture the correct trend in $\theta_{c}$ already within the employed model subset of $(2 \times 2)$ geometries.

It is interesting to notice that for the considered $4 d \mathrm{TM}$ sequence the computed $\theta_{c}$ for the initial $\mathrm{O}$ incorporation follows both in trend and approximate value the initially described $\theta_{c}^{\text {thd }}$, above which the bulk oxide phase becomes thermodynamically stable. We propose that this similarity of $\theta_{c}$ and $\theta_{c}^{\text {thd }}$ reflects an important bottleneck function of the initial $\mathrm{O}$ incorporation in the oxidation sequence of the considered surfaces: If $\theta_{c}$ was significantly smaller than $\theta_{c}^{\text {thd }}$, subsurface $\mathrm{O}$ could exist as a stable phase in the coverage range up to $\theta_{c}^{\text {thd }}$. This is apparently not the case, and $\mathrm{O}$ is incorporated only at coverages, where the bulk oxide is about to become stable. The close correlation of $\theta_{c}^{\text {thd }}$ with existing experimental data on the critical coverage for oxide formation $[4,20,21]$ then implies that at $\theta \approx$ $\theta_{c} \approx \theta_{c}^{\text {thd }}$ not only is $\mathrm{O}$ incorporated into the subsurface region, but this directly initiates the phase transition towards the formation of a surface oxide. Thus, the role of subsurface $\mathrm{O}$ is that of a metastable precursor, and at not too low temperatures the phase transition will proceed nearly instantaneously after the first incorporation of $\mathrm{O}$ below the surface.
With this interpretation, the initial incorporation of $\mathrm{O}$ may be used as a good measure to understand the ease of oxide formation of these TM surfaces. Setting out to analyze the obtained lowering of $\theta_{c}$ from $\operatorname{Ag}(111)$ to $\mathrm{Ru}(0001)$, we notice that surface structures containing both on-surface and subsurface $\mathrm{O}$ may be viewed as a "coadsorbate system," as the different sites available to the two types of $\mathrm{O}$ allow one to distinguish them as two species. In this spirit, the stability of these structures can be discussed in terms of the separate properties of the onsurface and subsurface $\mathrm{O}$, as well as the interaction between the two species. Within our simple model, the latter can be determined by comparing the $E_{b}$ computed for the mixed structures with the averaged $E_{b}$ of two calculations where $\mathrm{O}$ is present only in either the fcc or the tetra-I sites at the corresponding coverages. The values of this interaction energy are found to be rather small, i.e., $<0.2 \mathrm{eV}$, which is comprehensible as the $\mathrm{O}$ in tetra-I sites (located directly below the hcp sites) are rather distant to the onsurface $\mathrm{O}$ in fcc sites.

In the following discussion, we neglect this small interaction and analyze the initial subsurface incorporation of $\mathrm{O}$ just in terms of the separate stability of on-surface and subsurface $O$. Figure 3(a) shows the computed $E_{b}$ of
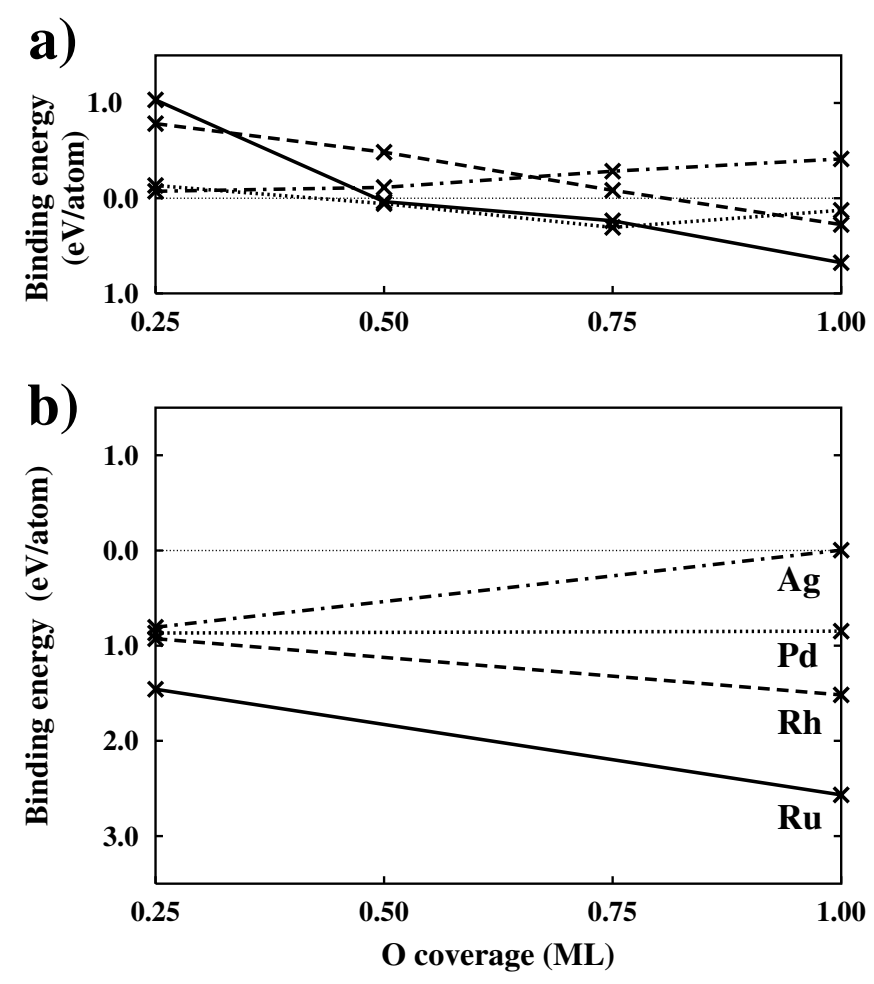

FIG. 3. Calculated $E_{b}$ for subsurface $\mathrm{O}$ incorporation into the tetra-I sites below the fully relaxed surface (no on-surface $\mathrm{O}$ present). The energy zero is the energy of a free $\mathrm{O}_{2}$, the clean surface at (a) its equilibrium geometry, and (b) at the (artificial) geometry of the respective adsorbate system (cf. text). $(\mathrm{Ru}=$ solid line, $\mathrm{Rh}=$ dashed line, $\mathrm{Pd}=$ dotted line, $\mathrm{Ag}=$ dash-dotted line). 
structures that contain from one $(\theta=0.25 \mathrm{ML})$ up to four $(\theta=1 \mathrm{ML})$ subsurface $\mathrm{O}$ in tetra-I sites. Considering the filling of the metallic $d$ band as the ruling quantity to determine the O-metal bond strength across the elements [13], one would typically expect decreasing $E_{b}$ from Ru to $\mathrm{Ag}$ as in the on-surface $\mathrm{O}$ chemisorption case depicted in Fig. 1. Interestingly, this is not the case, and, in particular, for $\mathrm{Ru}$ and $\mathrm{Rh}$ the energies are by several $\mathrm{eV}$ less favorable than for chemisorption in the (also highly coordinated) onsurface hollow sites.

The main reason behind this different behavior in Figs. 1 and 3(a) is the lattice distortion that the subsurface $\mathrm{O}$ needs to create. In all relaxed structures, the subsurface O-metal bond lengths are slightly larger than $2 \AA$. Yet, these Ometal bond lengths are incompatible with the available space inside a frozen metal lattice. For $\mathrm{Ru}$, which has the smallest lattice constant of the four considered materials, the tetra-I site would only allow for an O-metal bond length of $1.65 \AA$. This situation gradually becomes better for the other elements, yet for $\mathrm{Ag}$, which has the largest lattice constant, this value is with $1.80 \AA$ still significantly too short. Thus, subsurface $\mathrm{O}$ incorporation always induces a substantial local expansion of the metallic lattice [15,22]. The corresponding lattice deformation energy scales roughly as the bulk cohesive energy or the bulk modulus; i.e., it is largest for $\mathrm{Ru}$, where additionally the largest expansion is required, and smallest for Ag. This is reflected in the significantly increased $E_{b}$, particularly for $\mathrm{Ru}$ and $\mathrm{Rh}$ shown in Fig. 3(b), in which the cost of distorting the metal lattice has been approximately removed by computing these numbers with respect to a metal surface which had already been deformed into the final adsorption geometry.

The gradual decrease in the deformation cost from Ru to Ag all but compensates the $d$-band related weakening of the O-metal bond strength, resulting in the rather similar stability of subsurface $\mathrm{O}$ in all four elements. Because of this lattice distortion cost, subsurface $\mathrm{O}$ is in the low coverage limit always less stable than on-surface chemisorption. Yet, with increasing on-surface coverage, the repulsive interaction among the more densely packed adsorbates decreases the preference for on-surface adsorption; eventually $\mathrm{O}$ penetration may then become more favorable than a continued filling of the on-surface sites. Correspondingly, we find the critical total coverage, $\theta_{c}$, beyond which penetration starts to be lower, the lower the lattice deformation cost, i.e., it decreases from $\mathrm{Ru}$ to $\mathrm{Ag}$.

In conclusion, we have shown that $\mathrm{O}$ incorporation into the subsurface region commences at progressively lower coverages for the late $4 d \mathrm{TM}$ sequence from $\mathrm{Ru}$ to $\mathrm{Ag}$. The obtained coverages are very similar to those above which the oxide phase becomes already thermodynamically more stable than the chemisorbed $\mathrm{O}$ adlayer. We therefore propose that the role of subsurface $\mathrm{O}$ in the oxidation of these TM surfaces is that of a metastable precursor, such that the phase transition to the final oxide structure follows at not too low temperatures nearly instantaneously after incorporation of $\mathrm{O}$ below the surface becomes energetically favorable. As the stability of subsurface $\mathrm{O}$ was shown to depend strongly on the lattice deformation cost, this implies correlations between the oxidation behavior and properties such as the cohesive energy or bulk modulus. We acknowledge partial support by the DFG (Schwerpunkt "Katalyse").

*Present address: Institut für Chemie, Humboldt Universität zu Berlin.

[1] C. Stampfl et al., Surf. Sci. 500, 368 (2002), and references therein.

[2] H. Over et al., Science 287, 1474 (2000).

[3] B. L. M. Hendriksen and J.W.M. Frenken, Phys. Rev. Lett. 89, 046101 (2002).

[4] C. I. Carlisle et al., Surf. Sci. 470, 15 (2000), and references therein.

[5] J. P. Perdew, K. Burke, and M. Ernzerhof, Phys. Rev. Lett. 77, 3865 (1996).

[6] P. Blaha, K. Schwarz, and J. Luitz, WIEN97, TU Wien, Austria, 1999, ISBN 3-9501031-0-4.

[7] M. Bockstedte et al., Comput. Phys. Commun. 107, 187 (1997).

[8] FP-LAPW basis set: $R_{\mathrm{MT}}^{\mathrm{Ru}}=1.22 \AA, \quad R_{\mathrm{MT}}^{\mathrm{Rh}}=1.16 \AA$, $R_{\mathrm{MT}}^{\mathrm{Pd}}=1.19 \AA, R_{\mathrm{MT}}^{\mathrm{O}}=0.69 \AA, E_{\mathrm{max}}^{\mathrm{wf}}=17 \mathrm{Ry}, E_{\max }^{\mathrm{pot}}=$ $169 \mathrm{Ry}, 19 \mathbf{k}$ points in the irreducible $(1 \times 1)$ Brillouin zone (IBZ). The Troullier-Martins pseudopotentials employ $r_{s}=r_{p}=r_{d}=0.74 \AA$ for $\mathrm{O}$ [ $d$ as local potential], and $r_{s}=r_{d}=1.28$ and $r_{p}=1.39 \AA$ for $\mathrm{Ag}(s$ as local potential). $E_{\text {cut }}=50 \mathrm{Ry}, 21 \mathrm{k}$ points in the IBZ.

[9] C. Stampfl and M. Scheffler, Phys. Rev. B 54, 2868 (1996).

[10] M. V. Ganduglia-Pirovano and M. Scheffler, Phys. Rev. B 59, 15533 (1999); M. V. Ganduglia-Pirovano, K. Reuter, and M. Scheffler, Phys. Rev. B 65, 245426 (2002).

[11] K. Reuter et al., Chem. Phys. Lett. 352, 311 (2002); Phys. Rev. B 65, 165403 (2002).

[12] W. X. Li, C. Stampfl, and M. Scheffler, Phys. Rev. B 65, 075407 (2002).

[13] B. Hammer and J. K. Nørskov, Adv. Catal. 45, 71 (2001).

[14] Experimental heats of formation (eV/O atom): $\Delta H_{f}\left(\mathrm{RuO}_{2}\right)=1.59, \Delta H_{f}\left(\mathrm{Rh}_{2} \mathrm{O}_{3}\right)=1.19, \Delta H_{f}(\mathrm{PdO})=$ 0.88, $\Delta H_{f}\left(\mathrm{Ag}_{2} \mathrm{O}\right)=0.34$ [from CRC Handbook of Chemistry and Physics (CRC Press, Boca Raton, FL, 1995), 76th ed.].

[15] A. Kiejna and B. I. Lundqvist, Phys. Rev. B 63, 085405 (2001).

[16] J. Trost et al., J. Chem. Phys. 108, 1740 (1998).

[17] C. Stampfl et al., Phys. Rev. Lett. 77, 3371 (1996).

[18] K. D. Gibson et al., J. Chem. Phys. 110, 2757 (1999).

[19] J. Wider et al., Surf. Sci. 417, 301 (1998).

[20] E. Lundgren et al., Phys. Rev. Lett. 89, 246103 (2002).

[21] A. Böttcher and H. Niehus, J. Chem. Phys. 110, 3186 (1999).

[22] B. Chakraborty, S. Holloway, and J. K. Nørskov, Surf. Sci. 152, 660 (1985). 